\title{
Enhancing the photodynamic effect of hypericin in human bladder transitional cell carcinoma spheroids by the use of the oxygen carrier, perfluorodecalin
}

\author{
APPOLINARY R. KAMUHABWA ${ }^{1}$, ANN HUYGENS ${ }^{1}$, TANIA ROSKAMS $^{2}$ and PETER A.M. DE WITTE ${ }^{1}$ \\ ${ }^{1}$ Laboratorium voor Farmaceutische Biologie en Fytofarmacologie, Faculteit Farmaceutische Wetenschappen, \\ Katholieke Universiteit Leuven, Van Evenstraat $4 ;{ }^{2}$ Afdeling Histochemie en Cytochemie, Faculteit Geneeskunde, \\ Katholieke Universiteit Leuven, Minderbroederstraat 12, 3000 Leuven, Belgium
}

Received August 12, 2005; Accepted September 29, 2005

\begin{abstract}
In the present study, we evaluated the possibility of enhancing the photodynamic effect of hypericin in transitional cell carcinoma (TCC) spheroids by the use of the oxygen carrier, perfluorodecalin. Following incubation with hypericin, RT-112 TCC spheroids were irradiated in the presence or absence of perfluorodecalin, at light doses of $7 \mathrm{~J} / \mathrm{cm}^{2}$ or $28 \mathrm{~J} /$ $\mathrm{cm}^{2}$, delivered at a fluence rate of $15 \mathrm{~mW} / \mathrm{cm}^{2}$. The photodynamic therapy (PDT) efficacy was evaluated and apoptotic cells were visualized. The results show that, in the absence of perfluorodecalin, spheroidal TCC cells are inadequately sensitive to hypericin PDT. As was shown by fluorescence microscopy, this lack of activity was not due to insufficient photoactive concentrations of hypericin reaching the inner parts of the spheroids. Conversely, enhanced oxygenation of spheroids by perfluorodecalin led to a dramatic enhancement of hypericin PDT efficacy. The detection of nuclear shrinkage or fragmentation with DAPI staining and the assessment of cell morphology by light microscopy indicated that apoptosis was the most prominent response of spheroids to hypericin PDT in the presence of perfluorodecalin. In conclusion, the results of this study suggest that perfluorocarbons, such as
\end{abstract}

Correspondence to: Professor P. De Witte, Laboratorium voor Farmaceutische Biologie en Fytofarmacologie, Faculteit Farmaceutische Wetenschappen, Van Evenstraat 4, B-3000 Leuven, Belgium E-mail: peter.dewitte@pharm.kuleuven.ac.be

Abbreviations: BCG, Bacillus Calmette Guérin; CIS, carcinoma in situ; DAPI, 4',6-diamidino-2-phenylindole, dihydrochloride; $\mathrm{H} \& \mathrm{E}$, hematoxylin and eosin; MTT, 3-(4,5-dimethylthiazol-2-yl)2,5-diphenyl-tetrazolium bromide; PBS, phosphate-buffered saline; PD, perfluorodecalin; PDT, photodynamic therapy; TCC, transitional cell carcinoma

Key words: hypericin, RT-112 cells, photodynamic therapy, spheroids, perfluorodecalin perfluorodecalin, are useful in enhancing the oxygenation of tumor tissue, resulting in highly efficient hypericin PDT. Since hypericin becomes concentrated specifically in human bladder urothelial carcinoma lesions and the bladder is very well suited to instillation with a perfluorocarbon, combining the techniques looks very promising for an efficient and selective whole bladder wall photodynamic antitumoral treatment in a urological clinical setting.

\section{Introduction}

The treatment of superficial bladder tumors is based mainly on endoscopic resection combined with chemo- or immunotherapy by intravesical instillation. While the use of Bacillus Calmette Guérin (BCG) is likely to modify the recurrence profile of the disease, reduce the risk of progression and improve survival $(1,2)$, the resistance to BCG of certain bladder tumors and decrease of vesical compliance resulting from repetitive treatment remain therapeutic problems $(3,4)$.

Photodynamic therapy (PDT) has been suggested to be an alternative treatment for superficial bladder tumors. Transitional cell carcinoma (TCC) of the bladder can efficiently be treated with this type of therapy because, with careful control of the light distribution, curative doses can reach all parts of the urothelium (5-7). This is of importance in problematic superficial tumors, which are often multifocal. Areas of poorly defined dysplasia and carcinoma in situ (CIS) do not, therefore, necessarily need to be precisely defined for effective PDT to take place.

Hypericin, a hydroxylated phenanthroperylenequinone that is present in a number of plants of the genus Hypericum, becomes concentrated specifically in bladder urothelial carcinoma lesions after instillation in humans, and the compound is now used as a clinical diagnostic tool for the detection of superficial bladder tumors $(8,9)$. However, hypericin is also a photosensitizer with a potent photocytotoxic activity, indicating that hypericin could be used to treat bladder tumors photodynamically. Our preliminary in vitro and in vivo results with hypericin in the treatment of bladder tumors suggest that, although TCC tumor cells take up sufficient hypericin concentration, the spheroids (10) and the tumors (11) are not completely destroyed after light irradiation. 
Hypericin phototoxicity appears to be oxygen dependent (12) and mediated predominantly via the production of singlet oxygen (13), which indicates that there is oxygen consumption during light irradiation that renders tumor cells in the spheroids less sensitive to the photodynamic action of hypericin. This is supported by the fact that the PDT effects are decreased or abolished, both in vivo and in vitro, at low oxygen concentrations (14-16). There is evidence that, during PDT light irradiation, oxygen levels within the tumor are affected by the production of reactive oxygen species and PDT-induced vascular damage (17).

Perfluorocarbons are chemically inert in biological systems and have oxygen solubility coefficients of approximately $40-56 \mathrm{ml}$ oxygen per $100 \mathrm{ml}$ at 760 torr (18). Due to the high oxygen tension of perfluorocarbons, their administration can increase tissue oxygen tension. They dissolve approximately 20 -fold more oxygen than water or plasma. Since the demonstration of perfluorocarbons as oxygen carrying fluids for mice (19), numerous attempts have been made to use perfluorocarbons as an artificial blood substitute or to improve tissue oxygenation (20-22). Many perfluorocarbons are relatively non-toxic and, due to their high oxygen-carrying capacity, it is believed that these compounds can be used as oxygen carriers to enhance the PDT efficacy of photosensitizers.

Spheroids exhibit important features of avascular small tumors, such as CIS of the bladder, as well as sparsely vascularized tumors (23). Within spheroids, steep gradients can exist for cellular oxygen levels (24), proliferation (25), $\mathrm{pH}$ (26) and glucose concentration (27). Spheroids are, therefore, useful in studying some aspects involved in PDT, such as the role of oxygen and drug penetration. Since spheroid experiments are accomplished in vitro, this model facilitates studies of tumor cell-specific phenomena in isolation from complex host-dependent factors. In the present study, we investigated the enhancement of hypericin PDT efficacy by an oxygen carrier perfluorodecalin in multicellular human bladder RT-112 TCC spheroids.

\section{Materials and methods}

Preparation of hypericin solution. Hypericin was synthesized as reported previously (28). The stock solutions of hypericin were made in polyethylene glycol 400 and kept at $-20^{\circ} \mathrm{C}$ in the dark. Hypericin is insoluble in water and plasma proteins were used as an effective hypericin carrier for incubation with multicellular tumor spheroids. For this purpose, the stock solution was diluted in an aqueous buffer containing plasma proteins, as reported previously $(8,9)$.

Spheroid cultures. RT-112 human bladder TCC cells were obtained from the German Collection of Microorganisms and Cell Cultures (DSMZ, Braunschweig, Germany). Cells were grown at $37^{\circ} \mathrm{C}$ in humidified $5 \% \mathrm{CO}_{2}$ and $95 \%$ air atmosphere in minimum essential medium with Earle's Salt containing $2 \mathrm{mM}$ L-glutamine, $1 \%$ antibiotic/antimycotic solution, $1 \%$ non-essential amino acids and $10 \%$ fetal calf serum. The medium, cell culture ingredients and phosphate-buffered saline (PBS) were obtained from Gibco BRL, Paisley, Scotland.

Cell culture 96-well plates were underlayed with $1.5 \%$ agarose (Sigma, Steinheim, Germany). RT-112 spheroids were then initiated by plating $5 \times 10^{3}$ cells (per well) in $200 \mu 1$ of growth medium containing $1 \%$ sodium pyruvate $(100 \mu \mathrm{M})$ (Gibco BRL). The medium was replaced twice a week and the spheroids reached about $500 \mu \mathrm{m}$ in diameter after 7 days, at which point they were ready to use for experiments.

Intraspheroidal fluorescence distribution and quantification. Spheroids were incubated with hypericin $(5,30$ or $100 \mu \mathrm{M})$ in 24-well plates at $37^{\circ} \mathrm{C}$ for $2 \mathrm{~h}$. Afterwards, spheroids were immediately mounted in tissue Tek embedding medium (Miles, Elkhart, IN) and immersed in liquid nitrogen. Two consecutive $5-\mu \mathrm{m}$ frozen sections were centrally cut with a cryostat. The first section was examined by fluorescence microscopy and the second section was stained with hematoxylin and eosin (H\&E). Imaging of hypericin fluorescence in sections of spheroids was achieved by the use of fluorescence microscopy. The fluorescence microscope consisted of an Axioskop 2 plus fluorescence microscope (Carl Zeiss, Vision, Göttingen, Germany) illuminated by a $100 \mathrm{~W}$ mercury lamp. For fluorescence imaging, the filter set used included a 510 - to 560-nm band-pass excitation filter and a 590-nm long-pass emission filter. Fluorescence images were acquired using a light-sensitive charge-coupled device digital camera (AxioCam HR, Carl Zeiss, Göttingen, Germany).

A KS imaging software system (Carl Zeiss, Hallbergmoos, Germany) was used to measure the average fluorescence in concentric layers from the rim to the centre of the spheroid. The relative fluorescence intensity was then corrected for autofluorescence as measured on control spheroids that were not incubated with the photosensitizer. Fluorescence intensities corresponding to the relative intraspheroidal concentrations of hypericin were determined as the mean of eight spheroids.

Photodynamic treatment. Spheroids were incubated with hypericin $(5,30,100 \mu \mathrm{M})$ in 24 -well plates at $37^{\circ} \mathrm{C}$ for $2 \mathrm{~h}$. At the end of incubation, the hypericin solution was removed and replaced with PBS or perfluorodecalin (Sigma, Bornem, Belgium). Before application, perfluorodecalin was maximally oxygenated by bubbling oxygen through the liquid for $5 \mathrm{~min}$. For irradiation, light emitted by a Rhodamine $6 \mathrm{G}$ dye laser (375B, Spectra Physics, Mountain View, CA, USA) pumped by a $4 \mathrm{~W}$ Argon-laser (Spectra Physics) was coupled into a fiberoptic microlens (Rare Earth Medical, West Yarmouth, MA, USA) to obtain an ultra uniform intensity distribution. The laser was tuned at $595 \mathrm{~nm}$ and the laser beam was directed to the 24-well plate containing the spheroids. The light doses of $7 \mathrm{~J} / \mathrm{cm}^{2}$ and $28 \mathrm{~J} / \mathrm{cm}^{2}$ were delivered at a fluence rate of $15 \mathrm{~mW} / \mathrm{cm}^{2}$, as measured by an IL $1400 \mathrm{~A}$ photometer (International light, MA, USA).

The PDT efficacy was determined by the use of 3-(4,5dimethylthiazol-2-yl)-2,5-diphenyl-tetrazolium bromide (MTT; Sigma, St. Louis, MO, USA) dye reduction assay and by histological assessment with light microscopy. For the MTT antiproliferative assay, spheroids were trypsinized immediately after PDT at $37^{\circ} \mathrm{C}$. After centrifugation and vortexing, the cells from a given number of spheroids were counted and plated in $96-$ well plates $\left(1 \times 10^{4}\right.$ cells/well) at $37^{\circ} \mathrm{C}$ for $72 \mathrm{~h}$. Afterwards, MTT solution ( $1 \mathrm{mg} / \mathrm{ml}$ of PBS) was added to the cells and the plates were incubated at $37^{\circ} \mathrm{C}$ for $4 \mathrm{~h}$. The MTT was then removed and $100 \mu 1$ of dimethylsulfoxide was 

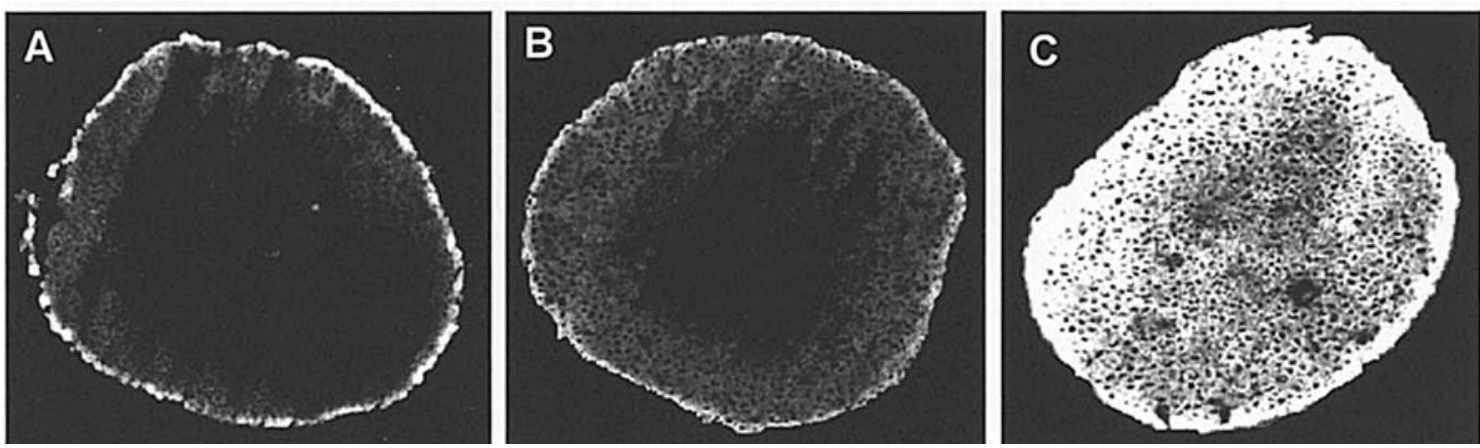

Figure 1. Fluorescence microphotographs of hypericin accumulation in the RT-112 TCC multicellular spheroids. Spheroids were incubated with $5 \mu \mathrm{M}$ (A), $30 \mu \mathrm{M}(\mathrm{B})$ or $100 \mu \mathrm{M}(\mathrm{C})$ hypericin for $2 \mathrm{~h}$. Frozen sections of spheroids were prepared as described in Materials and methods. Fluorescence microphotographs of the spheroid sections were acquired using fluorescence microscopy (magnification x400).

added to each well in order to dissolve the resulting formazan crystals. The plates were read on a microtitre plate reader (SLT, Salzburg, Austria) at an absorbance wavelength of $550 \mathrm{~nm}$. The light-induced cytotoxicity of different concentrations of hypericin in different PDT conditions with respect to the controls was determined after curve fitting using nonlinear regression (Prism, San Diego, CA, USA). The controls included spheroids that were not incubated with hypericin, but were irradiated in the presence of PBS or perfluorodecalin. All experiments were performed at least three times.

Histological assessment. For the histological evaluation of PDT efficacy, H\&E-stained sections of frozen spheroids were prepared as a function of time $(2,6,24$ and $72 \mathrm{~h})$ after PDT. The spheroid sections were then observed by light microscopy and the images were acquired using a lightsensitive charge-coupled device digital camera (AxioCam HR, Carl Zeiss, Göttingen, Germany).

Apoptosis detection. DAPI (4',6-diamidino-2-phenylindole, dihydrochloride) staining was used as nuclear staining to detect apoptotic nuclei. Centrally cut cryostat cross sections of spheroids were fixed in methanol-free formaldehyde (4\%) and, after washing with PBS, they were treated with $50 \mu \mathrm{l}$ Vectashield mounting medium (Vector Laboratories, Burlingame, CA) containing DAPI. Stained nuclei were visualized under a fluorescence microsocope using a 365/40-nm excitation filter and a 420-nm long-pass emission filter. Apoptotic cells were morphologically defined by nuclear shrinkage or fragmentation.

Statistical analysis. Statistically significant differences between values obtained under different experimental conditions were determined using the 2-tailed unpaired Student's t-test.

\section{Results}

Intraspheroidal fluorescence distribution. Fluorescence microphotographs of centrally cut frozen sections of hypericinexposed spheroids showed an overall good penetration of hypericin in the spheroids. Fig. 1 represents the fluorescence microphotographs of the RT-112 spheroids after incubation of the spheroids for $2 \mathrm{~h}$ with $5 \mu \mathrm{M}$ (Fig. 1A), $30 \mu \mathrm{M}$ (Fig. 1B) or $100 \mu \mathrm{M}$ (Fig. 1C) hypericin. The results show that the

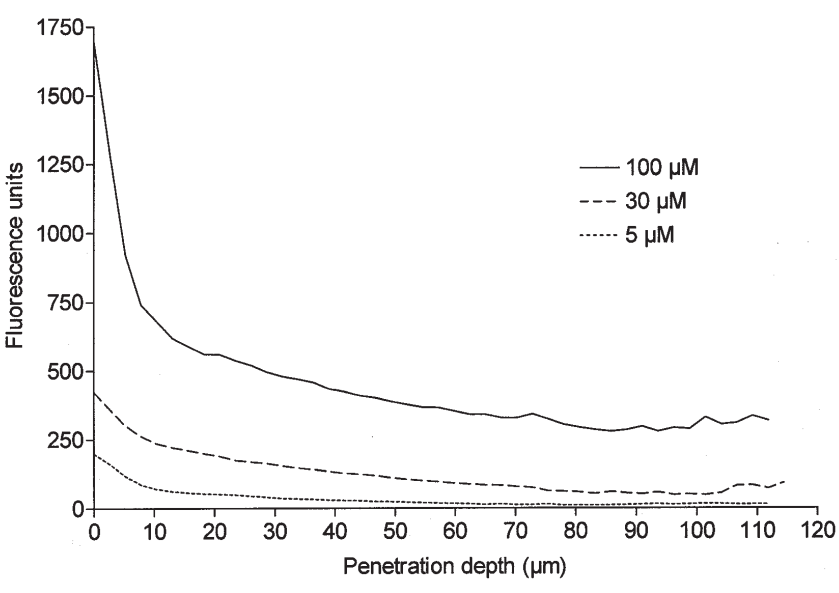

Figure 2. Quantification of the hypericin fluorescence in RT-112 TCC multicellular spheroids as function of the depth across the spheroid. Spheroids were incubated with 5,30 , or $100 \mu \mathrm{M}$ hypericin for $2 \mathrm{~h}$. Frozen sections of spheroids were prepared as described in Materials and methods. Fluorescence microphotographs of the spheroid sections were acquired using fluorescence microscopy and the average fluorescence in concentric layers of spheroids was determined. For each hypericin concentration the results are the average of 8 spheroids.

amounts of hypericin penetrating across the spheroid increased as a function of hypericin concentration. An imaging software system was used to measure the mean fluorescence from the rim to the central parts of the spheroids (Fig. 2). In all three concentrations used, a typical pattern can be seen with a high hypericin concentration at the periphery of the spheroid declining rapidly and reaching a steady state situation at about 70-100 $\mu \mathrm{m}$ below the surface. The highest concentration of $100 \mu \mathrm{M}$ hypericin induced substantially more fluorescence than the lower concentrations, both in the peripheral and central parts of the spheroid.

Cell survival. Using an MTT cell antiproliferative assay, the photocytotoxic effect of hypericin on the spheroids was investigated as a function of the photosensitizer concentration and light fluence. Fig. 3 presents the results of the cell survival after treatment of the spheroids with 5,30 or $100 \mu \mathrm{M}$ hypericin for $2 \mathrm{~h}$ and photo-activation with $7 \mathrm{~J} / \mathrm{cm}^{2}$ and $28 \mathrm{~J} / \mathrm{cm}^{2}$ light fluence delivered at $15 \mathrm{~mW} / \mathrm{cm}^{2}$ light fluence rate. The results 


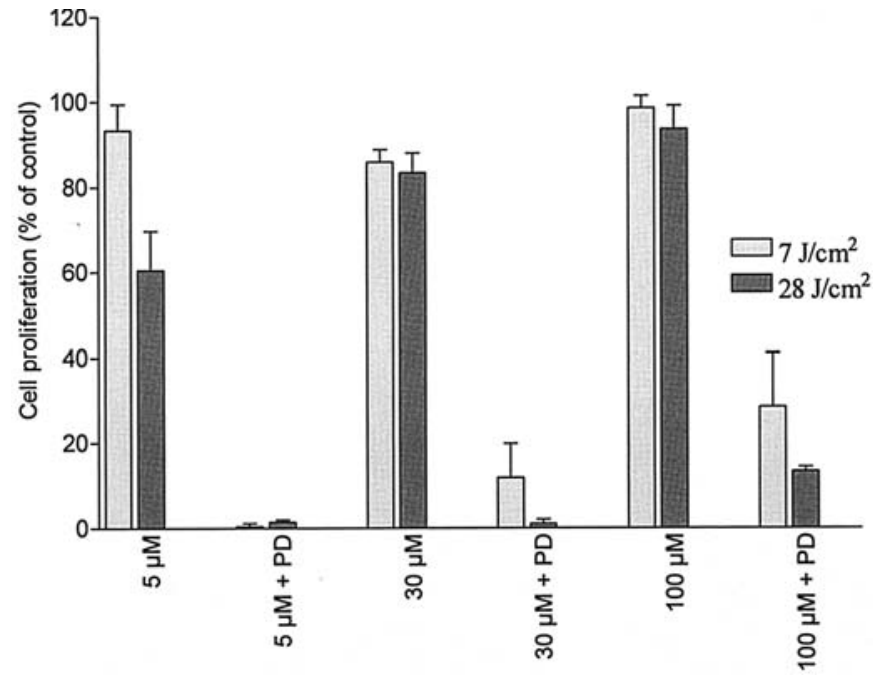

Figure 3. PDT of spheroids with or without perfluorodecalin. Spheroids were incubated with 5, 30, or $100 \mu \mathrm{M}$ hypericin for $2 \mathrm{~h}$. Afterwards, they were irradiated [in the presence of PBS or perfluorodecalin (PD)] with $7 \mathrm{~J} / \mathrm{cm}^{2}$ or $28 \mathrm{~J} / \mathrm{cm}^{2}$ laser light at the fluence rate of $15 \mathrm{~mW} / \mathrm{cm}^{2}$. After irradiation, spheroids were immediately dissociated by trypsinization and the surviving fraction of the cells was then determined using MTT assay as described in Materials and methods. Results are presented as surviving cells as the percentage of control (mean $\pm \mathrm{SD}, \mathrm{n}=3$ ).

are presented as percentage of cell survival of PDT-treated spheroids in comparison to controls.

After photodynamic treatment of the spheroids, the results show that, in the absence of perfluorodecalin, spheroidal cells are inadequately sensitive to hypericin PDT. However, enhanced oxygenation of spheroids using perfluorodecalin during light irradiation led to a dramatic enhancement of the hypericin PDT efficacy. For instance, hypericin PDT at $7 \mathrm{~J} / \mathrm{cm}^{2}$ using hypericin concentrations of 5, 30 and $100 \mu \mathrm{M}$ resulted in $93.3 \pm 6.15 \%, 85.8 \pm 2.9 \%$ and $97.8 \pm 2.9 \%$ (mean $\pm \mathrm{SD}, \mathrm{n}=3$ ) cell survival, respectively. Similar PDT conditions together with perfluorodecalin resulted in a dramatic decrease of cell survival; $0.48 \pm 0.71 \%, 11.9 \pm 7.9 \%$ and $28.4 \pm 12.61 \%$ (mean $\pm \mathrm{SD}, \mathrm{n}=3$ ) cell survival for hypericin concentrations of 5, 30 and $100 \mu \mathrm{M}$, respectively (Fig. 3). Intriguingly, the combination of hypericin PDT and perfluorodecalin was most effective with the lowest hypericin concentration (e.g.
Table I. PDT of spheroids with or without perfluorodecalin. ${ }^{a}$

\begin{tabular}{lll}
\hline & PDT & PDT + perfluorodecalin \\
\hline $5 \mu \mathrm{M}$ & $93.3 \pm 6.15$ & $0.48 \pm 0.71$ \\
$30 \mu \mathrm{M}$ & $85.8 \pm 2.9$ & $11.9 \pm 7.9$ \\
$100 \mu \mathrm{M}$ & $97.8 \pm 2.9$ & $28.4 \pm 12.61$ \\
\hline
\end{tabular}

2-tailed unpaired Student's t-test

$5 \mu \mathrm{M}$ PDT vs. $5 \mu \mathrm{M}$ PDT + perfluorodecalin

$30 \mu \mathrm{M}$ PDT vs. $30 \mu \mathrm{M}$ PDT + perfluorodecalin b

$100 \mu \mathrm{M}$ PDT vs. $100 \mu \mathrm{M}$ PDT + perfluorodecalin

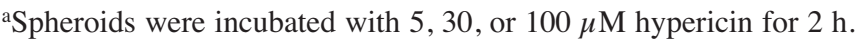
Afterwards, they were irradiated (in the presence of PBS or perfluorodecalin) with $7 \mathrm{~J} / \mathrm{cm}^{2}$ laser light at the fluence rate of $15 \mathrm{~mW} /$ $\mathrm{cm}^{2}$. After irradiation, spheroids were immediately dissociated by trypsinization and the surviving fraction of the cells was then determined with MTT assay as described in Materials and methods. Data are presented as surviving cells as the percentage of control (mean $\pm \mathrm{SD}, \mathrm{n}=3$ ). ${ }^{\mathrm{b}} \mathrm{p} 0.001$.

$5 \mu \mathrm{M}$ ) as compared to the higher concentrations (e.g. $100 \mu \mathrm{M}$ ) (Fig. 3 and Table I).

Histological evaluation. The results of the enhancement of hypericin PDT efficacy using perfluorodecalin were confirmed histologically using light microscopy (Fig. 4). The microscopic images obtained $24 \mathrm{~h}$ after light irradiation show that, in the case of PDT with perfluorodecalin, entire spheroids were destroyed (Fig. 4C). On the contrary, in the absence of perfluorodecalin (Fig. 4B), most of the spheroids were intact. Also, control spheroids which were irradiated in the presence of PBS or perfluorodecalin but in the absence of hypericin did not show structural alterations (Fig. 4A). At $72 \mathrm{~h}$ after PDT in the presence of perfluorodecalin, spheroids could no longer be detected, whereas spheroids that were photodynamically treated in the absence of perfluorodecalin were still intact (results not shown).
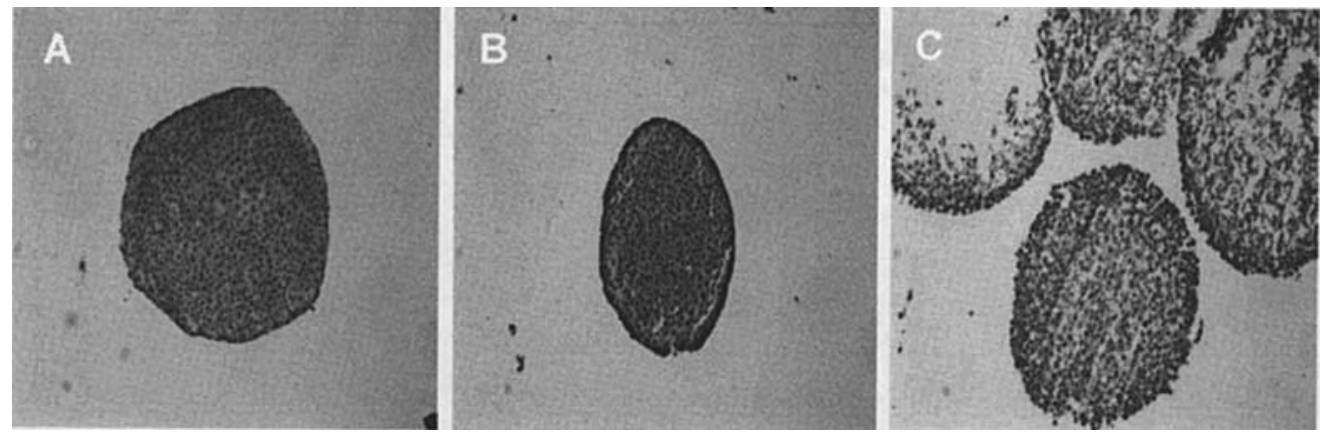

Figure 4. Light microscopy evaluation of the PDT effects of hypericin on the spheroids (magnification $\mathrm{x} 250$ ). Spheroids were incubated with $30 \mu \mathrm{M}$ hypericin for $2 \mathrm{~h}$. Afterwards, they were irradiated (in the presence of PBS or perfluorodecalin) with $7 \mathrm{~J} / \mathrm{cm}^{2}$ light at the fluence rate of $15 \mathrm{~mW} / \mathrm{cm}^{2}$. Frozen sections of spheroids were prepared, and microphotographs of H\&E-sections were acquired using light microscopy. (A) Control spheroids that were not incubated with hypericin but were irradiated in the presence of perfluorodecalin. (B) Spheroids that were incubated with hypericin and irradiated in the presence of PBS. (C) Spheroids that were incubated with hypericin and irradiated in the presence of perfluorodecalin. 

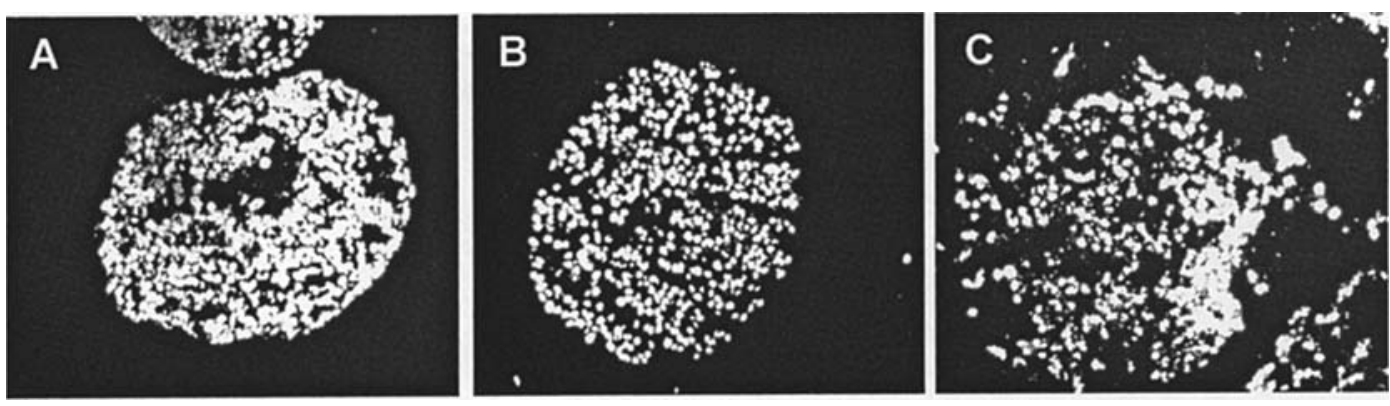

Figure 5. Fluorescence microphotographs of spheroids after staining for apoptosis detection. Apoptotic RT-112 cells in the spheroid sections were detected by DAPI staining. A, B and C represent the controls, PDT alone and PDT plus perfluorodecalin conditions, respectively. Only a few apoptotic cells could be observed in the case of hypericin PDT alone (B). There are no apoptotic cells in the control case (A) whereas hypericin PDT with perfluorodecalin resulted in apoptotic cell death (C).

Apoptosis detection. Apoptosis was determined by its characteristic morphological changes, including nuclear shrinkage and fragmentation. Nuclear changes in centrally cut cryostat cross sections of RT-112 spheroids were visualized after staining the nuclei with DAPI. The results are presented in Fig. 5. No apoptotic cell could be observed in the control conditions (Fig. 5A), whereas a few apoptotic cells were present in the case of hypericin PDT alone (Fig. 5B). On the contrary, most nuclei are apoptotic in the case of hypericin PDT with perfluorodecalin (Fig. 5C).

\section{Discussion}

As we reported previously, hypericin becomes concentrated specifically in bladder urothelial carcinoma lesions after instillation in humans $(8,9)$. Due to this specific localization of hypericin in urothelial carcinoma lesions, the compound is used nowadays as a clinical diagnostic tool for the detection of superficial bladder tumors. Since hypericin is also a photosensitizer with a potent photocytotoxic activity $(29,30)$, the specific location of hypericin in urothelial carcinoma lesions offers the unique opportunity to treat the tumor sites photodynamically.

Our previous in vitro (10) and in vivo (11) studies demonstrate that, although TCC tumor cells accumulate hypericin sufficiently, malignant cells are not completely destroyed after light irradiation. The 595-nm laser light used for photoactivation of hypericin penetrates readily across the $500-\mu \mathrm{m}$ spheroid. In addition, hypericin in combination with this light has been found to be effective in destroying tumors as large as $5 \mathrm{~mm}$ in diameter $(30,31)$. These data indicate that hypericin and light penetration through the spheroid are not limiting factors for hypericin PDT efficacy.

Based on the fact that hypericin phototoxicity is oxygen dependent (12) and is mediated predominantly via the production of singlet oxygen (13), it is believed that oxygen depletion during light irradiation renders tumor cells less sensitive to the direct photodynamic action of hypericin. It is suggested that the cause of low oxygen concentrations in spheroids is due to the static culture conditions as well as the PDT-induced hypoxia during light irradiation (32). The PDT-induced oxygen depletion, which results from photooxidation reactions, is so substantial that it only takes a few seconds for some cells in the treatment area to become anoxic, thus preventing further damage (32).
In the present study, we carried out experiments aimed at enhancing the oxygenation of TCC spheroids. As mentioned, numerous attempts have been made to use perfluorocarbons as an artificial blood substitute or to improve tissue oxygenation (20-22). Perfluorocarbons are also suggested to find a role in PDT by maintaining tissue oxygenation and thereby enhancing the PDT efficacy after light activation of the photosensitizer (33). Like many other perfluorocarbons, perfluorodecalin is known to dissolve oxygen efficiently [Dias et al, Proceedings 15th Symposium on Thermophysical Properties (www.symp15. nist.gov/sessions.htm), Bouldu, CO, 2003], an indication that this compound could be used as an oxygen carrier to enhance the PDT efficacy of photosensitizers.

The results explicitly demonstrate that only when spheroids are kept in enhanced oxygenation conditions by incubating them in perfluorodecalin during light irradiation, an adequate hypericin PDT response can be expected. The outcome therefore confirms the idea that hypericin is a potent photosensitizer that penetrates well through multiple cell layers reaching photo-active concentrations in the central parts in a 2-h period. However, the data equally prove that, in normal oxygenation conditions, insufficient oxygen diffuses into the tumoral tissue to compensate for the local oxygen consumption, resulting in a poor PDT response. Conversely, perfluorodecalin containing approximately 20 -fold more oxygen, as compared to PBS, creates a rich oxygen environment probably both in the spheroid and in its immediate environment. Of particular interest, cells present in spheroids incubated in perfluorodecalin did not shown any sign of cytotoxicity.

Interestingly, the combination of hypericin PDT and perfluorodecalin turned out to be most effective when a low hypericin concentration (e.g. $5 \mu \mathrm{M}$ ) was used. It is likely that the light activation of very high hypericin concentrations especially present at the outer parts of the spheroids, generates a zone in which all oxygen diffusing from the perfluorodecalin into the spheroids is converted into singlet oxygen. Hence, this oxygen-consuming barrier prevents deeper layers of the spheroid from being oxygenated adequately, making them insensitive to hypericin and light. The depletion of oxygen induced in these particular conditions therefore mimicks well the situation described for lower hypericin concentrations in normal oxygenation conditions.

The fact that cells in the spheroids that were treated with hypericin plus perfluorodecalin-PDT died through apoptosis was confirmed by the presence of apoptotic nuclei in the 
spheroid sections. Similarly to what was observed with histological assessment, hypericin PDT with perfluorodecalin induced cell apoptosis, even at $2 \mathrm{~h}$ after light irradiation. On the other hand, the DAPI staining results obtained with spheroids that were treated with hypericin PDT alone showed that only very few cells were apoptotic. Based on these results and on the results obtained by MTT assay and histological assessment, it is obvious that, in the case of PDT with perfluorodecalin, cells died through apoptosis whereas, in the case of PDT alone, most of the cells survived the PDT treatment. Hence, it appears that apoptosis rather than necrosis is a mechanism for hypericin plus perfluorodecalin PDTinduced cell death in RT-112 TCC spheroids.

In conclusion, the results of this study suggest that perfluorocarbons, such as perfluorodecalin, are useful in enhancing the oxygenation of tumor tissue, resulting in highly efficient hypericin PDT. Since hypericin becomes concentrated specifically in human bladder urothelial carcinoma lesions and the bladder is very well suited to instillation with a perfluorocarbon, combining the techniques looks very promising for an efficient and selective whole bladder wall photodynamic antitumoral treatment in a urological clinical setting.

\section{Acknowledgements}

We thank Ms. G. Luycks and her colleagues for their excellent technical support with the frozen sections of spheroids. This work was supported by grants awarded by 'Fonds voor Wetenschappelijk Onderzoek-Vlaanderen' (FWO Vlaanderen), the 'Onderzoeksfonds' of the K.U. Leuven (Onderzoekstoelage), and a 'Geconcerteerde Onder-zoeksactie' (GOA) from the Flemish Government. A. Kamuhabwa is a postdoc fellow supported by 'onderzoeksfonds K.U. Leuven' .

\section{References}

1. Herr HW, Pinsky CM, Whitmore WF, Sogani PC, Oettgen HF and Melamed MR: Long term effect of intravesical bacillus Calmette-Guérin on flat carcinoma in situ of the bladder. J Urol 135: 265-267, 1986.

2. Prout GR, Griffin PP and Daly JJ: The outcome of conservative treatment of carcinoma in situ of the bladder. J Urol 138: 766-770, 1987.

3. Lamm DL, Stogdill VD, Stogdill BJ and Crispen RG: Complications of bacillus Calmette-Guérin immunotherapy in 1,278 patients with bladder cancer. J Urol 135: 272-274, 1986.

4. Hudson MA: When intravesical measures fail. Indications for cystectomy in superficial disease. Urol Clin North Am 19: 601-609, 1992.

5. D'Hallewin MA, Baert L, Marijnissen JP and Star WM: Whole bladder wall photodynamic therapy with in situ light dosimetry for carcinoma in situ of the bladder. J Urol 148: 1152-1155, 1992.

6. Kriegmair M, Waidelich R, Lumper W, Ehsan A, Baumgartner R and Hofstetter A: Integral photodynamic treatment of refractory superficial bladder cancer. J Urol 154: 1339-1341, 1995.

7. Nseyo UO, DeHaven J and Dougherty TJ: Photodynamic therapy in the treatment of patients with resistant superficial bladder cancer: a long-term experience. J Clin Laser Med Surg 16: 61-68, 1998.

8. D'Hallewin MA, De Witte PA, Waelkens E, Merlevede W and Baert L: Fluorescence detection of flat bladder carcinoma in situ after intravesical instillation of hypericin. J Urol 164: 349-351, 2000 .
9. D'Hallewin MA, Kamuhabwa AR, Roskams T, De Witte PAM and Baert L: Hypericin-based fluorescence diagnosis of bladder carcinoma. BJU Int 89: 760-763, 2002.

10. Kamuhabwa AR, Huygens A and De Witte PAM: Photodynamic therapy of transitional cell carcinoma multicellular tumor spheroids with hypericin. Int J Oncol 23: 1445-1450, 2003.

11. Kamuhabwa A, Roskams T, D'Hallewin M, Baert L, Van Poppel $\mathrm{H}$ and De Witte P: Whole bladder wall photodynamic therapy of orthotopic transitional cell carcinoma bladder tumors using intravesically administered hypericin. Int J Cancer 107: 460-467, 2003.

12. Delaey E, Vandenbogaerde A, Merlevede W and De Witte P: Photocytotoxicity of hypericin in normoxic and hypoxic conditions. J Photochem Photobiol B Biol 56: 19-24, 2000.

13. Thomas $C$ and Pardini RS: Oxygen dependence of hypericininduced photocytotoxicity to EMT6 mouse mammary carcinoma cells. Photochem Photobiol 55: 831-837, 1992.

14. Gomer CJ and Razum NJ: Acute skin response in albino mice following porphyrin photosensitization under oxic and anoxic conditions. Photochem Photobiol 40: 435-439, 1984.

15. Henderson CM and Dougherty TJ: How does photodynamic therapy work? Photochem Photobiol 55: 145-157, 1992.

16. Dougherty JT and Marcus SL: Photodynamic therapy. Eur J Cancer 28A: 1734-1742, 1992.

17. Fuchs $\mathrm{J}$ and Thiele J: The role of oxygen in cutaneous photodynamic therapy. Free Radic Biol Med 24: 835-847, 1998.

18. Wilhelm E and Battino R: Thermodynamic functions of the solubilities of gases in liquids at $25^{\circ} \mathrm{C}$. Chem Rev 73: 1-9, 1977.

19. Clark LC and Gollan F: Survival of mammals breathing organic liquids equilibrated with oxygen at atmospheric pressure. Science 152: 1755-1756, 1966.

20. Millard RW: Oxygen solubility, rheology and hemodynamics of perfluorocarbon emulsion blood substitutes. Artif Cells Blood Substit Immobil Technol 22: 235-244, 1994.

21. Riess JG and Le Blan M: Solubility and transport phenomena in perfluorochemicals relevant to blood substitution and other biomedical applications. Pure Appl Chem 54: 2383-2392, 1982.

22. Dracker RA: The development and use of oxygen carrying blood substitutes. Immunol Invest 24: 403-410, 1995.

23. Hamilton G: Multicellular spheroids as an in vitro tumor model. Cancer Lett 131: 29-34, 1998.

24. Carlson J and Acker H: Influence of the oxygen pressure in the culture medium on different types of multicellular spheroids. Int J Radiat Oncol Biol Phys 11: 535-546, 1985.

25. Allison DC, Yuhas JM, Ridolpho PF, Anderson SL and Johnson TS: Cytophotometric measurement of the cellular DNA content of $(3 \mathrm{H})$ thymidine-labelled spheroids. Cell Tissue Kinet 16: 237-246, 1983 .

26. Acker H, Carlsson J, Holterman G, Nederman T and Nylen T: Influence of glucose and buffer capacity in the culture medium on growth and $\mathrm{pH}$ in spheroids of human thyroid carcinoma and human glioma origin. Cancer Res 47: 3504-3508, 1987.

27. Li CKN: The glucose distribution in 9L rat brain multicell tumor spheroids and its effect on cell necrosis. Cancer 50: 2066-2073, 1982.

28. Falk H, Meyer J and Oberreiter M: A convenient semisynthetic route to hypericin. Monatsh Chem 124: 339-341, 1993.

29. Zupkó I, Kamuhabwa A, D'Hallewin M, Baert L and De Witte P: In vivo photodynamic therapy of hypericin in transitional cell carcinoma bladder tumors. Int J Oncol 18: 1099-1105, 2001.

30. Kamuhabwa A, Agostinis P, D'Hallewin M, Baert L and De Witte P: Cellular photodestruction induced by hypericin in AY-27 rat bladder carcinoma cells. Photochem Photobiol 74: 126-132, 2001.

31. Chen B and De Witte P: Photodynamic therapy efficacy and tissue biodistribution of hypericin in mouse P388 lymphoma tumor model. Cancer Lett 150: 111-117, 2000.

32. Foster TH, Hartley DF, Nichols MG and Hilf R: Fluence rate effects in photodynamic therapy of multicell tumor spheroids. Cancer Res 53: 1249-1254, 1993.

33. Chowdhary RP, Cundall RB and Morgan CG: Influence of fluorocarbon emulsions on porphyrin-sensitised oxidation of histidine. Photochem Photobiol 51: 395-400, 1990. 